\title{
Structural and frequency dependencies of a.c. and dielectric characterizations of epitaxial InSb-based heterojunctions
}

\author{
A ASHERY ${ }^{1}$, A H ZAKI ${ }^{2}$, M HUSSIEN MOURAD ${ }^{3}$, A M AZAB ${ }^{4}$ and A A M FARAG ${ }^{5,6, *}$ \\ ${ }^{1}$ Solid State Physics Department, Physics Division, National Research Center, Dokki, Giza 12311, Egypt \\ ${ }^{2}$ Electronics Department, Military Technical College, Cairo 11371, Egypt \\ ${ }^{3}$ Department of Engineering Applications of Laser (E.A.L), National Institute of Laser Enhanced Sciences (N.I.L.E.S), \\ Cairo University, Giza 12613, Egypt \\ ${ }^{4}$ Solid State Electronics Laboratory, Solid State Physics Department, Physics Division, \\ National Research Center, Dokki, Giza 12311, Egypt \\ ${ }^{5}$ Department of Physics, Faculty of Science and Arts, Aljouf University, Aljouf 75911, Saudi Arabia \\ ${ }^{6}$ Thin Film Laboratory, Department of Physics, Faculty of Education, Ain Shams University, \\ Roxy, Cairo 11757, Egypt
}

MS received 11 July 2015; accepted 8 February 2016

\begin{abstract}
In this work, heterojunction of InSb/InP was grown by liquid phase epitaxy (LPE). Surface morphology and crystalline structure of the heterojunction were characterized by scanning electron microscopy (SEM) and $\mathrm{X}$-ray diffraction (XRD). The frequency and temperature dependences of a.c. conductivity and dielectric properties of the heterojunctions were investigated in the ranges of $100 \mathrm{kHz}-5 \mathrm{MHz}$ and $298-628 \mathrm{~K}$, respectively. The a.c. conductivity and its frequency exponents were interpreted in terms of correlated barrier hopping model (CBH), as the dominant conduction mechanism for charge carrier transport. The calculated activation energy, from the Arrhenius plot, was found to decrease with increasing frequency. Experimental results of both dielectric constant $\varepsilon_{1}$ and dielectric $\operatorname{loss} \varepsilon_{2}$ showed a remarkable dependence of both frequency and temperature.
\end{abstract}

Keywords. Liquid phase epitaxy; InSb/InP; a.c. conductivity; dielectric constants.

\section{Introduction}

Recently it was found that III-V compound semiconductors provide the materials suitable for electronic and optoelectronic devices [1,2]. Indium antimonide (InSb)-based devices, as an example for this type of group, has its applications in high-electron-mobility and heterostructure bipolar transistors, diode lasers, light-emitting diodes, photodetectors and electro-optic modulators [1]. Moreover, InSb has a significant amount of interest due to its narrow direct band gap and smallest electron effective mass $[1,2]$. These characteristics make InSb to access for some more important applications such as infrared detection arrays, high speed electronics and magnetoresistive sensors $[1,3]$.

Sophisticated epitaxial growth techniques are valid for a wide range of material systems like III-V compound semiconductors, such as molecular beam epitaxy (MBE), metal organic chemical vapour deposition (MOCVD) and liquid phase epitaxy (LPE) have been successfully implemented for the growth process that are widely used for a variety of electronic and optoelectronic devices [4,5].

Ravikant Sharma et al [6] have fabricated InP on $\mathrm{InSb}$ strained heterojunctions by MOCVD and studied the current

\footnotetext{
*Author for correspondence (alaafaragg@gmail.com, alaafaragg2014@hotmail.com)
}

transport properties. They found a high strain limits for the transport of carriers and overlapping for the depletion regions.

Benamara et al [7] have investigated the effect of surface preparation and annealing on the electrical parameters of $\mathrm{Au} / \mathrm{Indium}$ Phosphide (InP) and Au/InSb/InP Schottky diodes. They have obtained a high-quality Schottky-type contact with elevated barrier height values of about $0.63 \mathrm{eV}$. Moreover, Akkal et al [8] have performed the characterization of the interface states in $\mathrm{Au} /$ heated $\operatorname{InSb} / \mathrm{InP}(100)$ Schottky diode and the determination of the energy density distribution and relaxation time of the interface. They also calculated the density of interface states and relaxation time using capacitance measured at different frequencies. Chellalia et al [9] have obtained high-quality Schottky type contact with elevated barrier height value of about $0.501 \mathrm{eV}$. They explained the improvement by the passivation of the substrate, using $\mathrm{Sb}$ atoms, which prevent any migration of the semiconductor components during the alumina and gold deposition. Ferrera et al [10] have presented the self-organization of InSb-capped quantum dots grown by atomic layer molecular beam epitaxy (ALMBE). They also studied the evolution of island morphology as the InSb amount is increased by transmission electron microscopy (TEM). Moreover, they discussed the effect of the introduction of the cap layer by Raman spectroscopy in terms of TEM results. 
To the best of our knowledge, there are little reports on the growth of InSb/InP heterojunction device by LPE [11-13]. Moreover, there are no enough efforts concerning the conduction mechanisms and dielectric properties of such structure. The aim of the present work is to study the morphological and structural characteristics of such heterojunction. Moreover, the effect of temperature and applied frequencies on electrical conductivity for $\mathrm{InSb} / \mathrm{InP}$ in a frequency range 100 $\mathrm{kHz}-5 \mathrm{MHz}$ and a range of temperature 298-628 K were also considered. In addition, the dielectric properties of such heterojunction have also been discussed.

\section{Experimental}

\subsection{Fabrication of InSb/InP heterojunction by LPE}

LPE technique was employed to grow layers of InSb on InP substrate using indium as a solvent. The multibin boat made of special graphite hardness is held at a fixed position within a silica tube, and a thermocouple is fixed under the boat. The system is usually evacuated to $10^{-2} \mathrm{~Pa}$. Prior to the growth run, purified argon is passed through the tube. The details for the used LPE technique is described elsewhere [11]. The boat was loaded with single-crystalline InSb substrates. The growth solution contained indium with InSb pieces. The loaded boat was heated up to $650 \mathrm{~K}$ and kept at this temperature for $30 \mathrm{~min}$ to homogenize the solution and then cooled down with a cooling rate of $0.5 \mathrm{~K} \mathrm{~min}^{-1}$. The obtained heterojunctions were coated from the back side (InP side) by thin films of Au-ohmic contact using the thermal evaporation technique, and then the InSb layer was overcoated by an Ag metal to be used as ohmic contact. These contacts has a low resistance with each material and have a lower barrier which can be vanished as compared to the barrier originated from $\mathrm{InSb} / \mathrm{InP}$ interface. The area of above contact is about $1 \mathrm{~cm}^{2}$ and the thickness of the metal contact in each case is about $100 \mathrm{~nm}$ as determined by the thickness monitor [13]. The heterojunction characteristics were checked using current-voltage characteristics of InSb/InP. An exponential increase in the forward current with applied voltage at low voltage range was obtained, not shown here. This result is similar to the results obtained by our group worker elsewhere for similar structure and can be attributed to the formation of depletion region between $\mathrm{InSb}$ and InP thin films [11].

\subsection{Characterization techniques}

Scanning electron microscopy (SEM) type REMMA-202 was used for examination of the surface morphology of the heterojunction with accelerating voltage up to $20 \mathrm{kV}$, resolution can attain about $70 \AA$ and magnification ranges are 10-400000×.

The crystalline structure of the heterojunction was examined using X-ray diffraction (XRD) analysis. The XRD pattern was recorded using an X-ray diffractometer (X'PERT PRO, PNA analytical Inc.) with a $\operatorname{CuK}_{\alpha}(\lambda=1.5418 \AA)$

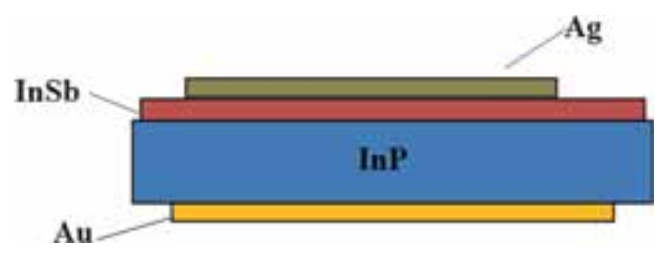

Figure 1. Schematic diagram of $\mathrm{InSb} / \mathrm{InP}$ heterojunction.

radiation produced at an applied voltage and beam current of $25 \mathrm{kV}, 15 \mathrm{~mA}$, respectively. The XRD spectrum was acquired for $2 \theta$ in the range of $20-90^{\circ}$ at continuous scan of $1 \mathrm{~s}$ for step size of $0.01^{\circ}$.

The a.c. conductivity as well as dielectric measurements were carried out by the aid of a programmable automatic RLC bridge, model Hioki 3532-50 LCR HiTester, in the temperature range of 298-628 K and the frequency range of 100 $\mathrm{kHz}-5 \mathrm{MHz}$. The a.c. conductivity is considered to be across the junction through the barrier at the heterojunction interface, i.e., current flows across $\mathrm{p}-\mathrm{n}$ boundary. The temperature of the heterojunction was tested using chromel-alumel thermocouple over the entire temperature range. The schematic diagram of the front view of $\mathrm{InSb} / \mathrm{InP}$ heterojunction is shown in figure 1.

\section{Results and discussion}

\subsection{Morphology and crystalline structure characterization}

Figure $2 \mathrm{a}$ and $\mathrm{b}$ shows the surface morphology of the $\mathrm{InSb} /$ InP heterojunction using SEM at different magnifications of 3000 and $5000 \times$. As shown, a highly smooth surface and a more homogenous morphology of epitaxial InP are the characters of the obtained structure. Moreover, a uniform grain size distribution and grain size are considered to be low and cannot be obviously resolved. The only feature evident is dust-like particles resting on the surface. Furthermore, the microscopic findings of the smooth surface confirmed its polished appearance and the suitability of the prepared heterojunction for optoelectronic applications.

The crystalline structure of the epitaxially grown InSb layer onto InP substrate was examined by using XRD and shown in figure $3 \mathrm{a}$. Obviously, the sharp peak at around $2 \theta \approx$ $30.4^{\circ}$, is the characteristic peak of single crystalline InP of preferred (200) orientation, in accordance with JCPDS card no. (03-065-0320). The additional peak of a preferred (400) orientation is the characteristic for InSb with tetragonal structure according to JCPDS card no. 03-065-0320). The mean crystallite size (mean crystallite diameter) was calculated using the full-width at half-maximum (FWHM) of (400) peak from Scherrers formula as follows [14]:

$$
D=\frac{K \lambda}{\beta \cos \theta},
$$

where $K$ is the shape factor (related to the crystal shape), $\lambda$ the $\mathrm{X}$-ray wavelength of $\mathrm{CuK}_{\alpha}$ radiation, $\theta$ the Braggs angle 

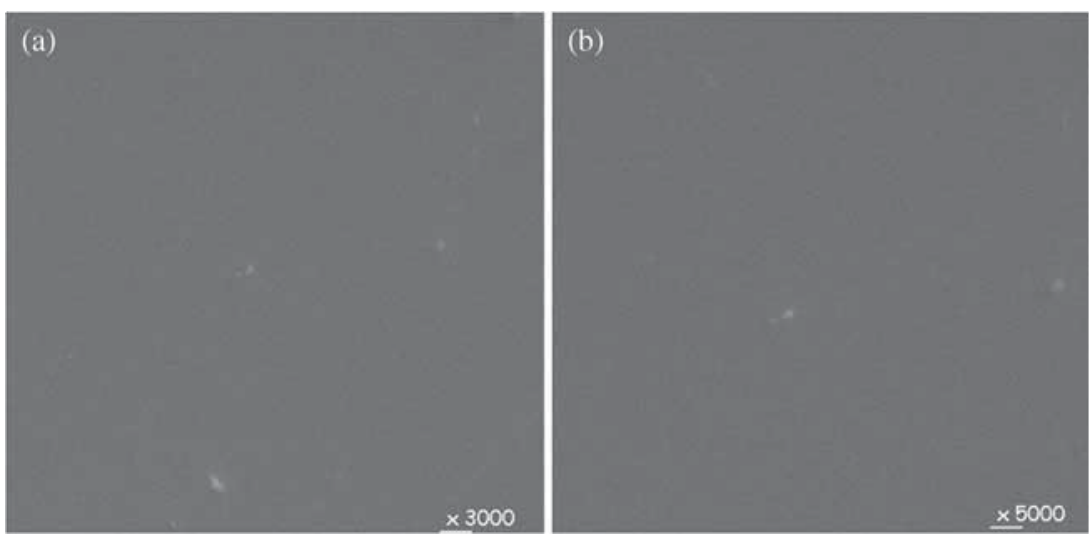

Figure 2. Top view of SEM image of InSb/InP heterojunction.
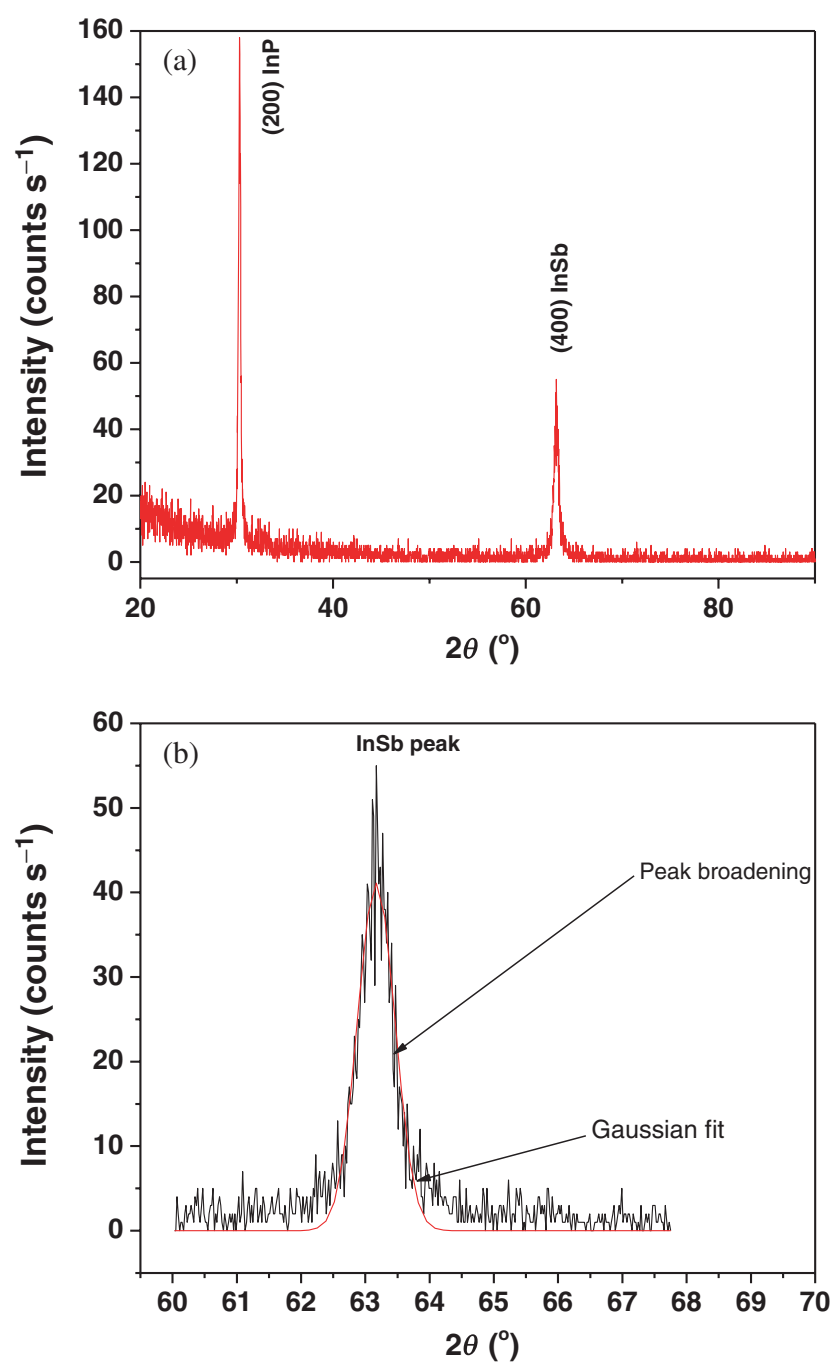

Figure 3. (a) XRD of InSb/InP heterojunction. (b) Peak broadening and Gaussian fitting of InSb epilayer.

and $\beta$ the full-width at half-maximum of the peak with instrument corrections. The mean crystalline size of the InSb layer were determined by the X-ray line broadening method using the above equation. It is well known that the width of the
Table 1. The main XRD parameters of InSb.

\begin{tabular}{ccccc}
\hline Film & $D(\mathrm{~nm})$ & $\varepsilon \times 10^{-4}$ & $\delta \times 10^{15} \mathrm{~m}^{-2}$ & $N \times 10^{17} \mathrm{~m}^{-3}$ \\
\hline InSb & 21 & 2 & 2.3 & 2.16 \\
\hline
\end{tabular}

Bragg's peak is considered to be an incorporation of both instrument- and sample-dependent effects. To separate these contributions, the collection of the diffraction pattern from the line broadening of a standard material such as silicon was made to determine the instrumental broadening. Moreover, the broadening of the InSb peak with Gaussian fitting is shown in figure $3 b$ to estimate the mean crystallite size and other related parameters. The mean crystallite size was calculated and listed in table 1 . The obtained value of the mean crystallite size of InSb epilayer gives an evidence for the nanostructure characteristic of the obtained layer. The other microstructural parameters such as the lattice microstrain $\left(\varepsilon^{*}\right)$ and dislocation density $(\delta)$ and the number of crystallites per unit area $(N)$ and $t$ the film thickness, can be calculated using the relations [14],

$$
\begin{aligned}
& \varepsilon^{*}=\frac{\beta \cos \theta}{4}, \\
& \delta=\frac{1}{D^{2}}, \\
& N=\frac{t}{D^{3}} .
\end{aligned}
$$

These microstructural parameters were determined depending on the broadening and Gaussian fit of the InSb peak and listed in table 1. Moreover, the film thickess can be obtained from the FWHM as discussed before in the literature [15].

\subsection{Frequency and temperature dependencies of a.c. conductivity}

Figure $4 \mathrm{a}$ shows the frequency dependence of the a.c. conductivity $\left(\sigma_{\text {a.c. }}\right)$ for $\mathrm{InSb} / \mathrm{InP}$ heterojunctions in the frequency 

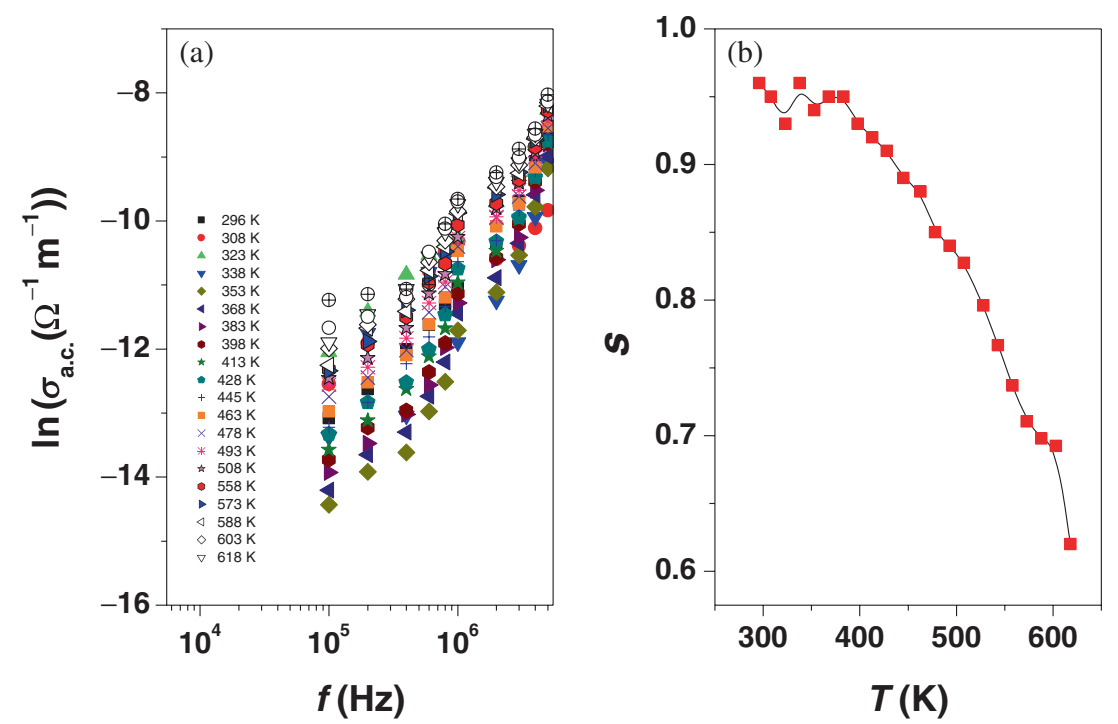

Figure 4. (a) Frequency dependence of a.c. conductivity at different temperatures of $\mathrm{InSb} / \mathrm{InP}$ heterojunction. (b) Temperature dependence of frequency exponent of $\mathrm{InSb} / \mathrm{InP}$ heterojunction.

range of $200 \mathrm{kHz}-5 \mathrm{MHz}$ at different measuring temperatures in the range of $298-628 \mathrm{~K}$. It is evident from this figure that $\sigma_{\text {a.c. }}$ determines observable frequency dependence through the desired frequency range which suggests that hopping conduction is a predominant and also the increase in the applied frequency enhances the hopping of charge carriers between the localized states [16]. The a.c. conductivity $\sigma_{\text {a.c. }}(\omega)$ can be expressed by the following relation $[17,18]$ :

$$
\sigma_{\text {a.c. }}(\omega)=B(\omega)^{\mathrm{s}},
$$

where $B$ is a constant and $s$ the frequency exponent parameter which depends on the temperature and can be used for the determination of a.c. conduction mechanism.

The frequency exponent, $s$ can easily be obtained from the slopes of linear part of the relation of $\ln \sigma_{\text {a.c. }}(\omega)=f(\omega)$ at different temperatures. The temperature dependence of $s$ for the investigated heterojunction is shown in figure $4 \mathrm{~b}$. It is clear from this figure that $s$ decreases as the temperature increases. Various theoretical models [19] based on the relaxation characteristics proceeded by the hopping or tunnelling of charge carriers between equilibrium sites were sophisticated to elucidate the frequency and temperature dependences of a.c. conductivity and the frequency exponent, $s$ [19].

One of the main predominant conduction mechanisms is the correlated barrier hopping $(\mathrm{CBH})$ model, in which the charge carrier hops between sites over the potential barrier separating them. Moreover, the value of $s$, is temperaturedependent and decreases as the temperature increases i.e., $s$ tends to be unity as the temperature tends to $0 \mathrm{~K}$. This result reveals that $\mathrm{CBH}$ model is the most appropriate to characterize the a.c. electrical conduction for the studied heterojunction and in the desired temperature range. For this model, the frequency exponent, $s$ is given by the following expression [17]:

$$
s=1-\frac{6 k T}{\left[W_{\mathrm{m}}+k T \ln \left(\omega \tau_{0}\right)\right]} .
$$

A first approximation of the above equation gives the simple expression for the frequency exponent $s$ as follows:

$$
s=1-\frac{6 k T}{W_{\mathrm{m}}} .
$$

The average value of $W_{\mathrm{m}}$ is obtained and found to be $0.62 \mathrm{eV}$ at room temperature $(298 \mathrm{~K})$. This value is approximately a quarter of the optical band gap of InP thin films (1.96 eV [20]), which indicates that the single polaron hopping is the dominating conduction mechanism in such films $[21,22]$.

Temperature dependence of $\sigma_{\text {a.c. }}$ of the heterojunction is shown in figure $5 \mathrm{a}$ at certain frequencies. As observed, $\sigma_{\text {a.c. }}$ increases linearly by increasing the temperature. The temperature dependence of $\sigma_{\text {a.c. }}$ is stronger at low frequencies than at relatively high frequencies. This suggests that $\sigma_{\text {a.c. }}$ is a thermally activated process from different localized states in the gap [23]. The relation exhibits two distinct regions with different slopes. The dependence of $\sigma_{\text {a.c. }}$ on temperature usually fitted to obey Arrhenius equation expressed by

$$
\sigma=\sigma_{0} \exp \left(\frac{-\Delta E}{k T}\right)
$$

where $\sigma_{0}$ is the pre-exponential factor, $\Delta E$ the activation energy for the thermally activated process, $k$ the Boltzmann's constant and $T$ the absolute temperature. 
Activation energy of a.c. conduction, $\Delta E$ was calculated at different frequencies from the slopes of the linear fit of figure $5 \mathrm{~b}$. Obviously, $\Delta E$ is found to decrease with the increase in frequency. Lower values of the obtained activation energy confirm the applicability of hopping conduction as a dominant current transport mechanism in our heterojunction. Furthermore, the increase of the applied frequency enhances the electronic jumps between the localized states [24].

Dependence of the real part of dielectric constant, $\varepsilon_{1}$ on the temperature and frequency of $\mathrm{InSb} / \mathrm{InP}$ heterojuntion is shown in figure $6 \mathrm{a}$ and $\mathrm{b}$. Figure $6 \mathrm{a}$ shows the frequency dependence of the dielectric constant $\left(\varepsilon_{1}\right)$ for the heterojunction at different constant temperatures in the range of 298-628 K. As observed, $\varepsilon_{1}$ is found to decrease with increasing frequency. The higher value of $\varepsilon_{1}$ at low frequencies is due to the contribution of multi-component of polarization mechanisms such as electronic, ionic, orientation and interface [25]. For further increase in frequency, the dipoles cannot rotate sufficiently and rapidly, so that their oscillations lag behind those of the field. Later, the dipole will be completely unable to follow the field and the orientation polarization is nearly stopped and then only the interfacial polarization exists. Figure $6 \mathrm{~b}$ shows the temperature dependence of the dielectric constant $\varepsilon_{1}$ at different frequencies. In addition, $\varepsilon_{1}$
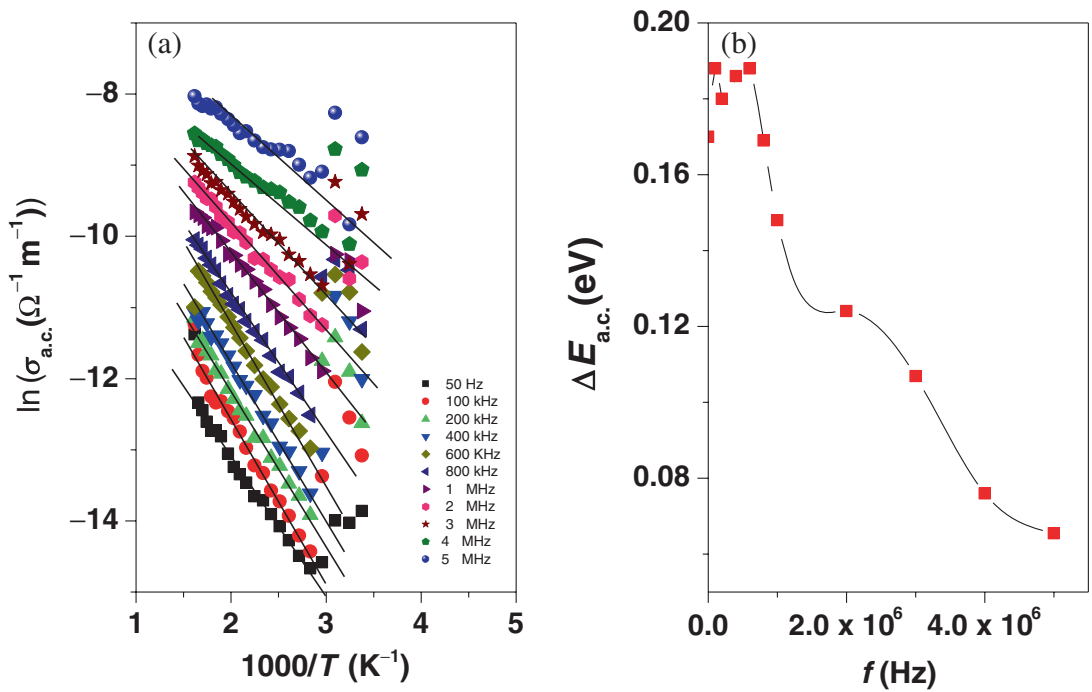

Figure 5. (a) Temperature dependence of a.c. conductivity at different temperatures of InSb/InP heterojunction. (b) Frequency dependence of activation energy of InSb/InP heterojunction.
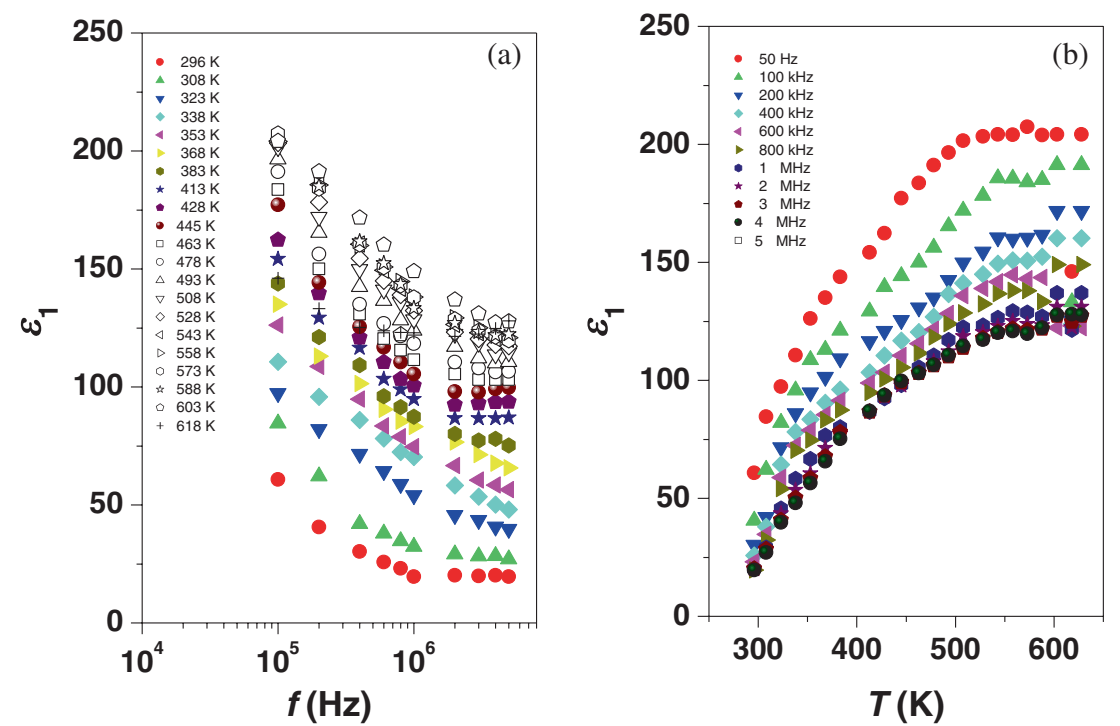

Figure 6. (a) Frequency dependence of real dielectric constant at different temperatures of InSb/InP heterojunction. (b) Temperature dependence of real dielectric constant of $\mathrm{InSb} / \mathrm{InP}$ heterojunction. 
increases as the temperature increases over the desired frequency range. The increase of $\varepsilon_{1}$ with increase in temperature can be attributed to the fact that dipoles cannot orient themselves at low temperatures. When the temperature increases, the orientation of the dipoles is available and thus increases the orientational polarization, and consequently $\varepsilon_{1}$ increases.

Frequency dependence of dielectric loss, $\varepsilon_{2}$ for the InSb/ InP heterojunction at different temperatures in the range of $298-628 \mathrm{~K}$ is shown in figure 7a. Obviously, $\varepsilon_{2}$ decreases with increasing the applied frequency. This behaviour can be explained by the fact that the value of $\varepsilon_{2}$, at low frequencies, is attributed to the migration of ions in the material. At moderate frequencies $\varepsilon_{2}$ is due to the contribution of ions jump, conduction loss of ions migration and ions polarization loss. At high frequency, ion vibrations may be the only source of dielectric loss and so $\varepsilon_{2}$ has the minimum value [26].

Temperature dependence of $\varepsilon_{2}$ for the InSb/InP heterojunction at some fixed frequencies in the range of $100 \mathrm{kHz}-5$ $\mathrm{MHz}$ is shown in figure $7 \mathrm{~b}$. As observed, $\varepsilon_{2}$ exhibits strong temperature dependence at higher temperatures and lower frequencies, increases with increasing temperature. Increase of $\varepsilon_{2}$ with increase in temperature can be explained by the
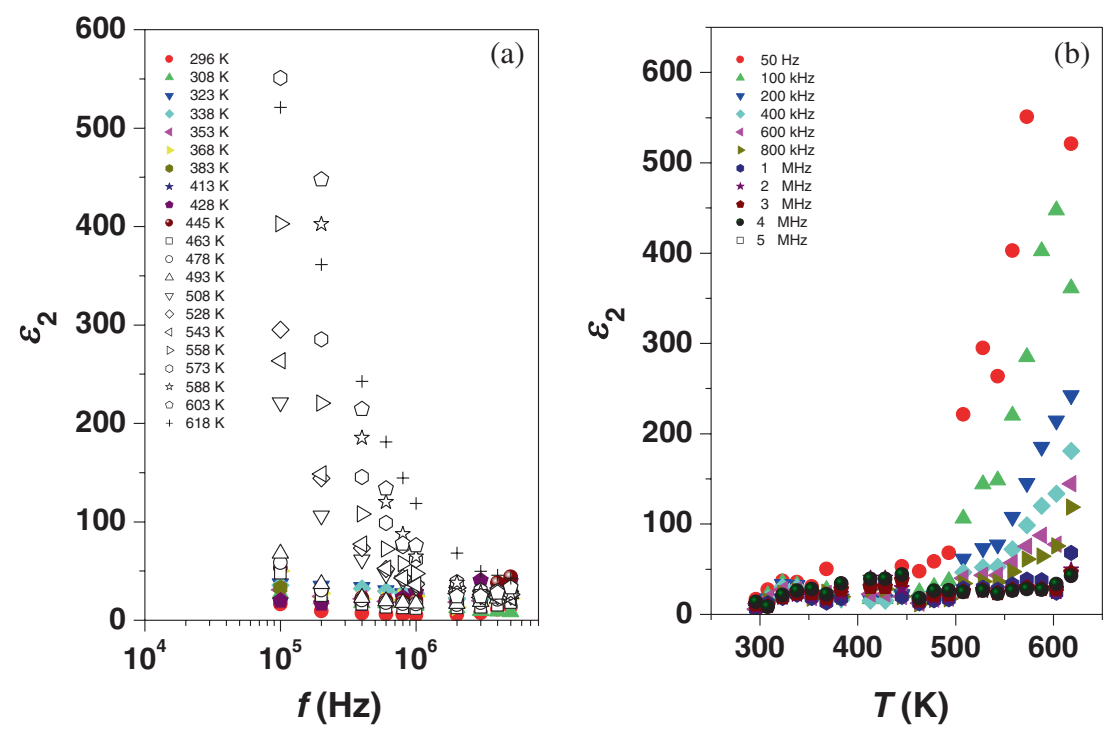

Figure 7. (a) Frequency dependence of imaginary dielectric constant at different temperatures of $\mathrm{InSb} / \mathrm{InP}$ heterojunction. (b) Temperature dependence of imaginary dielectric constant of InSb/InP heterojunction.
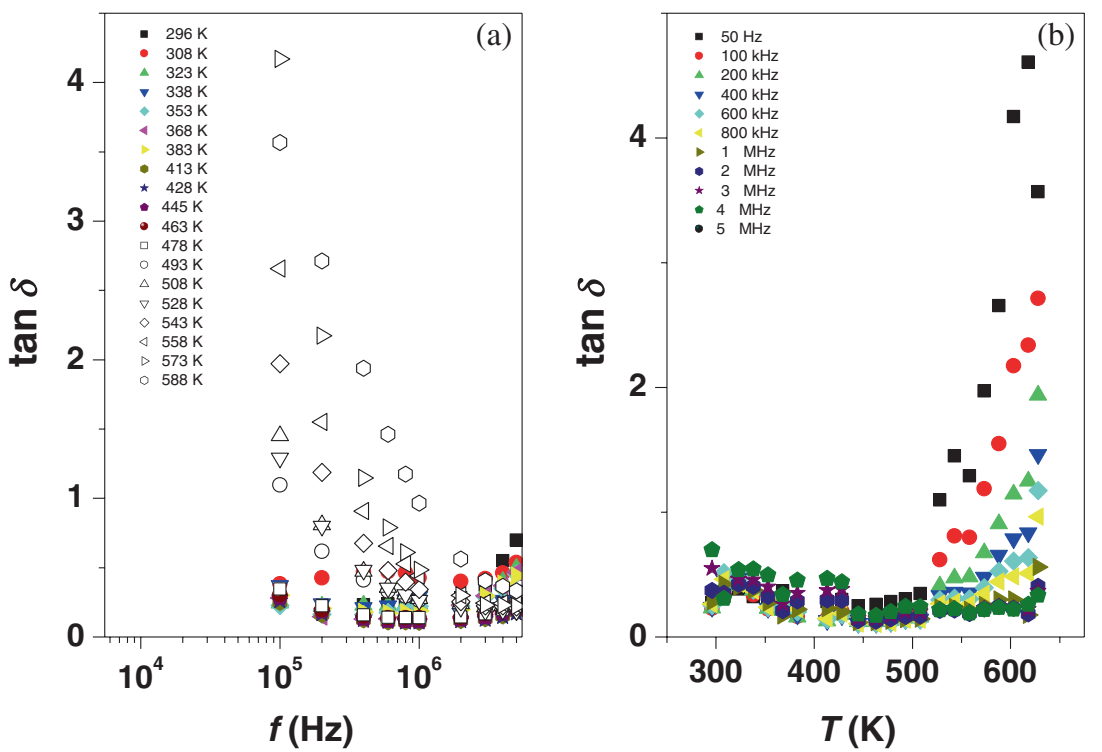

Figure 8. (a) Frequency dependence of dielectric loss at different temperatures of InSb/InP heterojunction. (b) Temperature dependence of dielectric loss constant of $\mathrm{InSb} / \mathrm{InP}$ heterojunction. 
fact that dipoles cannot be oriented at low temperatures (remain frozen), while at high temperatures, dipoles can rotate freely as suggested [27].

Variation of the dielectric loss $(\tan \delta)$ against frequency and temperature is shown in figure 8a. As shown in this figure, the value of $\tan \delta$ decreases with the increasing frequency in the temperature range of 298-628 K. Dielectric loss occurs when the electric polarization in a dielectric material is unable to follow the variation of a.c. field that alter the energy difference which produces a net polarization [28]. Moreover, figure $8 \mathrm{~b}$ shows the dielectric loss $(\tan \delta)$ against temperature at different frequencies. As observed, value of $\tan \delta$ increases with increase in temperature in the desired temperature range.

\section{Conclusions}

Morphology and crystalline structural parameters like mean crystallite size, microstrain, dislocation density and the number of crystallites/unit area of InSb/InP heterojunction grown by LPE were achieved. The a.c. conductivity is found to increase with increasing frequency and follows a universal power law $\left(\sigma_{\text {a.c. }}=A \omega^{\mathrm{s}}\right)$ and the frequency exponent, $s$ decreases with increasing temperature, which signifies that the $\mathrm{CBH}$ is the dominant charge transport mechanism. Application of $\mathrm{CBH}$ model reveals that electronic conduction takes place by single polaron depending on the calculated binding energy. The dielectric constant, dielectric loss and dielectric tangent of the heterojunction were found to decrease with increasing frequency and increase with increasing temperature. The increase of both $\varepsilon_{1}(\omega)$ and $\varepsilon_{2}(\omega)$ towards the low frequency region may be attributed to interfacial polarization that occurs via space charges formed in the heterojunction.

\section{Acknowledgements}

This work was carried out through the collaboration among Solid State Physics Department, National Research Center, Egypt; Department of Engineering Applications of Laser, National Institute of Laser Enhanced Sciences, Cairo University, Egypt; Electronics Department, Military Technical College, Egypt; and Department of Physics, Faculty of Education, Ain Shams University, Egypt.

\section{References}

[1] Yadav A D, Rao B V, Dubey S K and Gadkari D B 2001 Mater. Sci. Eng. B 84176
[2] Mukhin M S, Terent'ev Ya V, Golub L E, Nestoklon M O, Meltser Ya B and Semenov A N et al 2011 Acta Phys. Pol. A 120868

[3] Terent'ev Ya V, Toropov A A, Meltser B Y, Semenov A N, Solov'ev V A, Sedova I V et al 2010 Semiconductors 44194

[4] Suprem R D, Collin J D, Dmitri Z, Yong P C, Timothy D S and David B J 2011 Appl. Phys. Lett. 98243504

[5] Ashley T, Buckle L, Datta S, Emeny M T, Hayes D G, Hilton $\mathrm{K} P$ et al 2007 Electron. Lett. 43777

[6] Sharma R, Paul B and Banerji P 2010 Appl. Surf. Sci. 256 2232

[7] Benamara Z, Akkal B, Talbi A, Gruzza B and Bideux L 2002 Mater. Sci. Eng. C 21287

[8] Akkal B, Benamara Z, Gruzza B, Bideux L and Bouiadjra N B 2002 Mater. Sci. Eng. C 21291

[9] Chellalia M, Akkala B, Tizia S, Benamara Z, Gruzza B, Robert C and Bideux L 2000 Mater. Sci. Eng. B 7719

[10] Ferrera J C, Peiróa F, Corneta A, Morantea J R, Utzmeierc T and Armellesc G 1998 Microelectron. Eng. 4351

[11] Farag A A M, Terra F S, Ashery A and Mansour A M 2014 J. Alloys Compd. $\mathbf{6 1 5} 604$

[12] Dixit K, Rodrigues B V, Bhat H L, Venkataraghavan R, Chandrasekaran K S and Arora B M 2002 J. Cryst. Growth 235154

[13] Baca A G, Ren F, Zolper J C, Briggs R D and Pearton S J 1997 Thin Solid Films 308599

[14] Kumaran T S and Banu S P 2013 Int. J. Recent Sci. Res. 4168

[15] Ayers J A 2007 Heteroepitaxy of semiconductors (USA: CRC Press and Taylor \& Francis Group)

[16] Chen R H, Chang R Y and Shern C S 2006 Solid State Ionics 1772857

[17] Elliott S R 1987 Adv. Phys. 36135

[18] Ben Rhaiem A, Hlel F, Guidara K and Gargouri M 2009 J. Alloys Compd. $\mathbf{4 8 5} 718$

[19] El-Menyawy E M, Zeyada H M and El-Nahass M M 2010 Solid State Sci. 122182

[20] Gayen R N, Das S N, Dalui S, Paul R, Bhar R and Pal A K 2010 Thin Solid Films $\mathbf{5 1 8} 3595$

[21] Dakhel A A 2006 J. Alloys Compd. 4221

[22] Dakhel A A 2008 Curr. Appl. Phys. 8134

[23] El-Nahass M M, Ali H A M, Saadeldin M and Zaghllol M 2012 Physica B $\mathbf{4 0 7} 4453$

[24] El-Nahass M M and Ali H A M 2012 Solid State Commun. 1521084

[25] Dakhel A A 2005 Solid State Electron 491996

[26] El-Nahass M M, Atta A A, El-Zaidia E F M, Farag A A M and Ammar A H 2014 Mater. Chem. Phys. 143490

[27] Shekharam T, Rao V L, Yellaiah G, Kumar T M and Nagabhushanam M 2014 J. Alloys Compd. 617952

[28] Louati B, Hlel F and Guidara K 2009 J. Alloys Compd. 486 299 